\title{
Influence of Idiopathic Pulmonary Fibrosis Progression on Healthcare Resource Use
}

\author{
Alex Diamantopoulos ${ }^{1}$ (1) - Toby M. Maher ${ }^{2,3} \cdot$ Nils Schoof $^{4} \cdot$ Dirk Esser $^{4}$. \\ Corinne LeReun ${ }^{5}$
}

Published online: 25 June 2018

(C) The Author(s) 2018

\begin{abstract}
Background Disease progression and acute exacerbations in patients with idiopathic pulmonary fibrosis (IPF) are associated with high morbidity and mortality. They usually require a visit to a specialist or a general practitioner (GP) in less severe cases or hospitalisation in more severe cases. Objective The objective of this study was to identify factors that influence resource use in IPF.

Methods Clinical and healthcare resource use data were collected in two large, international, multi-centre, randomised controlled trials (RCTs) that studied nintedanib for the treatment of IPF (INPULSIS-1 and -2). The pooled data of nintedanib and placebo included 1014 patients followed for 12 months. The trial data were analysed in 3-month intervals. We studied two dependent variables: the occurrence of allcause hospitalisation and visits to a physician (GP or specialist). The independent variables included the change in forced vital capacity percent predicted (FVC\% $\%$ pred), investigator-reported acute exacerbation events, age, time since diagnosis, smoking status, and sex.

Results Hospitalisation during a 3-month interval was significantly associated with a drop of at least 5 or 10 points in FVC\%pred (odds ratios [ORs] $1.58[p=0.009]$ and 2.62
\end{abstract}

Alex Diamantopoulos

alexd@symmetron.net

1 Symmetron Limited, Kinetic Centre, Theobald Street, Elstree, Herts WD6 4PJ, UK

2 NIHR Biomedical Research Unit Royal Brompton Hospital, London, UK

3 Fibrosis Research Group, National Heart and Lung Institute, Imperial College London, London, UK

4 Boehringer Ingelheim GmbH, Ingelheim, Germany

5 Independent Statistician, Sainte-Anne, Guadeloupe, France $[p<0.001])$ and associated with the occurrence of at least one acute exacerbation (OR 14.44; $p<0.001$ ) during the same interval. The above factors remained significant when repeating the analysis for hospitalisation based on change in FVC\%pred or events occurring during the previous 3 months interval. Smoker status and a unit change in $\mathrm{FVC} \%$ pred during the previous interval were added to the significant factors. Physician visits during a 3-month interval were significantly associated with a lower FVC\%pred at the start of the interval (per 10-point decrement, OR 1.05; $p=0.040$ ) and with the change in FVC\%pred during the same interval (per 10 -point loss, OR 1.13; $p=0.042$ ). Visits were also associated with a 5-point drop in FVC\%pred (OR 1.23; $p=0.020$ ), age (per 5-year increments OR 1.07; $p=0.028$ ), and female $\operatorname{sex}(\mathrm{OR} 1.32 ; p=0.017)$. Nevertheless, the predictive power of the models was considered poor for both outcomes (hospitalisation and physician visits).

Conclusions Disease progression and acute exacerbation events are significantly associated with hospitalisation of patients with IPF. Outpatient visits to physicians are associated with disease progression, baseline FVC\% \%red, age and sex.

\section{Key Points for Decision Makers}

The analysis conducted was conceived with the intention to be useful for future economic evaluations based on economic modelling.

Disease progression was associated with an increase in both occurrence of hospitalisation and physician visits.

Acute exacerbation events were associated with more hospitalisations but not visits to a physician. 


\section{Introduction}

Idiopathic pulmonary fibrosis (IPF) is a fatal lung disease involving progressive dyspnoea and loss of lung function [1]. More than 5000 new cases occur annually in the UK alone [2], with an incidence of three to nine cases per 100,000 per year in Europe and North America [3]. The disease rarely occurs in people aged $<45$ years. Approximately two-thirds of patients with IPF are current or former smokers; in fact, the disease often co-occurs with chronic obstructive pulmonary disease [2]. Pathogenesis may involve aberrant signalling by tyrosine kinases such as vascular endothelial growth factor (VEGF), fibroblast growth factor (FGF) and platelet-derived growth factor (PDGF) in alveolar epithelial cells and interstitial fibroblasts [4]. This aberrant signalling leads to proliferation of fibroblasts with laying down of extra-cellular matrix and subsequent tissue remodelling.

Only two drugs are currently licensed to treat IPF: pirfenidone (Esbriet, Roche) was approved by the European Medicines Agency (EMA) in 2011 and the US FDA in 2014, and nintedanib (OFEV, Boehringer Ingelheim) was approved by the EMA in 2015 and the FDA in 2014 [5-7]. Both drugs work by slowing progression, without curing the disease. In animal models, pirfenidone was shown to inhibit fibrosis by inhibiting fibroblast proliferation and reducing production of fibrogenic signaling molecules such as transforming growth factor (TGF)- $\beta$, which in turn reduces TGF- $\beta$-stimulated collagen production [8]. Pirfenidone also inhibits inflammatory processes associated with IPF by reducing the production of inflammatory mediators such as tumor necrosis factor (TNF)- $\alpha$ and interleukin (IL)-1 $\beta$. Nintedanib is a specific tyrosine kinase inhibitor, also targeting PDGF, FGF and VEGF receptors. Nintedanib was shown to inhibit fibroblast proliferation, migration, contraction and differentiation to the active extracellular matrix-secreting myofibroblast $[9,10]$. Nintedanib has also demonstrated to have anti-inflammatory activities by reducing inflammatory mediators IL- $1 \beta$ and IL-6. A double-blind, placebo-controlled phase II randomised controlled trial (RCT) involving 432 patients with IPF suggested that 12-month treatment with nintedanib was associated with smaller decline in forced vital capacity (FVC), fewer acute exacerbations, and maintenance of health-related quality of life [8]. Two replicate, placebocontrolled phase III RCTs (INPULSIS-1, INPULSIS-2) involving 1066 patients confirmed that nintedanib slows the rate of decline in FVC, indicating slower disease progression [9].

In addition to slowing IPF progression, nintedanib helps prevent acute exacerbations, defined as rapid worsening of disease in the absence of infection, heart failure, pulmonary embolism or other identifiable cause [10]. Acute exacerbations are associated with high morbidity and mortality [11-13]. They usually require a visit to a specialist or a general practitioner (GP) in less severe cases or hospitalisation in more severe cases.

It is estimated that patients with IPF live for a median of 3 years after diagnosis $[2,14]$. The probability of survival beyond 5 years is $20-30 \%[2,15]$. This implies potentially substantial healthcare resource use extending over several years as the disease progresses and patients experience acute exacerbations. It follows that it is crucial to understand how healthcare resources are affected by IPF progression and related acute exacerbations. One common method of analysing the burden of a disease is to study claims data and combine it with patient costs. This method often provides information at a macro level, without exploring specific factors that influence the changes in healthcare resources. The present study takes a micro approach, using evidence from clinical trials to analyse factors influencing hospitalisation and visits to physicians. This approach and the analysis conducted was conceived with the intention to be useful for future economic evaluations based on economic modelling.

\section{Methods}

Data for this retrospective analysis were taken from the INPULSIS-1 and INPULSIS-2 RCTs [9]. Patients were eligible to participate in these trials if they were aged $\geq 40$ years, had been diagnosed with IPF within the previous 5 years, had FVC percent predicted (FVC\%pred) $>$ $50 \%$, had lung diffusion capacity for carbon monoxide $\left(\mathrm{DL}_{\mathrm{co}}\right)$ that was $30-79 \%$ of the predicted value, and had undergone high-resolution computed tomography (HRCT) of the chest within the previous 12 months. Patients were randomised to receive nintedanib $150 \mathrm{mg}$ or placebo twice daily (bid) for 52 weeks with the option to interrupt treatment or reduce the dose to $100 \mathrm{mg}$ bid to manage adverse events.

To improve precision of our estimates, data for patients in the nintedanib and placebo arms were analysed together, since we were focused on the disease impact rather than specific treatment effects. Patient data used included age at baseline, sex, time since IPF diagnosis at baseline, and smoking status at baseline (current smoker, ex-smoker, never smoker). In the clinical trial, FVC was measured and FVC\% \%red calculated at least every 3 months, therefore the following clinical data were analysed over four 3-month intervals: FVC\%pred at the start of the interval, absolute change in $\mathrm{FVC} \%$ pred during the interval, and occurrence of investigator-reported acute exacerbation during the interval (current-interval analysis). The analysis 

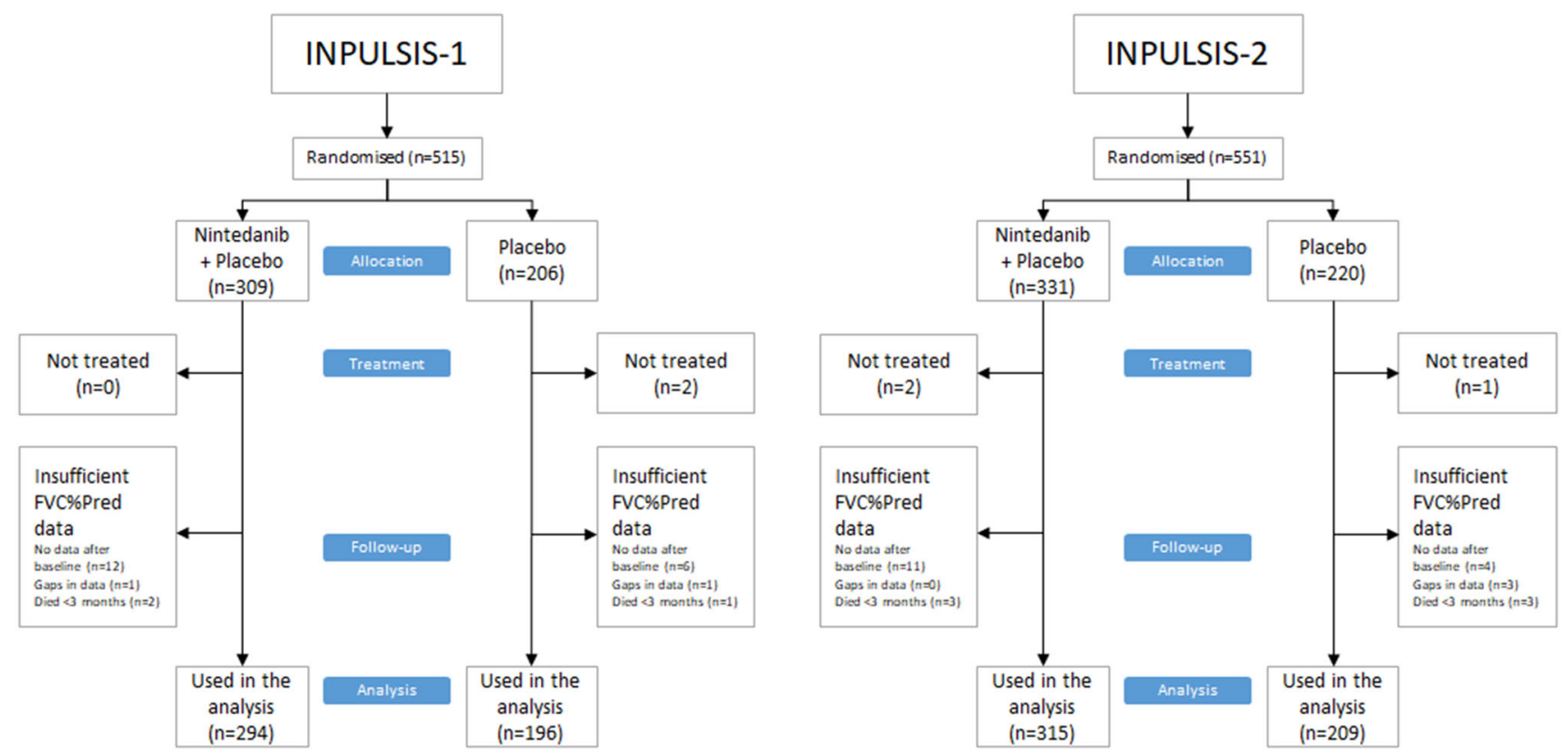

Fig. 1 Patients in the analysis from the two INPULSIS trials FVC\%pred force vital capacity percent predicted

was repeated for the absolute FVC\%pred, a change in FVC\%pred, and an exacerbation event during the previous 3-month interval (previous-interval analysis).

Acute exacerbation was defined as an event meeting all the following criteria, based on investigator assessment [9]: unexplained worsening or development of dyspnoea within the previous 30 days; new diffuse pulmonary infiltrates observed on chest radiography and/or HRCT, or development of parenchymal abnormalities in the absence of pneumothorax or pleural effusion (new ground-glass opacities) since the previous visit; and exclusion of known causes of acute worsening, including infection, left heart failure, pulmonary embolism, and any identifiable cause of acute lung injury. Unscheduled inpatient and outpatient visits were recorded retrospectively during each study visit on a healthcare resource utilisation electronic case report form. Inpatient visits were further consolidated with information from the serious adverse event forms (i.e. serious adverse events requiring a hospitalisation).

\subsection{Outcomes}

The primary outcomes of interest were whether, at least once during a given 3-month interval, a patient was hospitalised for any reason ${ }^{1}$ and whether a patient visited a physician (GP or specialist) for any reason. ${ }^{2}$ As a secondary outcome, the intensity of the resource use was

\footnotetext{
${ }^{1}$ Question in the report form: "Has the patient been hospitalised since the last trial visit?"

${ }^{2}$ Question in the report form: "Has the patient had any unscheduled outpatient or home visit since the last trial visit?"
}

analysed: the total duration of hospitalisation(s) (in days) during a given 3-month interval and the total number of visits to a physician during the interval. The analysis included only the unscheduled visits to a hospital or a physician; study per-protocol visits were not considered.

\subsection{Statistical Analysis}

All analyses were performed in STATA 14 [16] using a significance threshold of 5 and $10 \%$ as sensitivity analysis. Analyses of primary outcomes were based on data from INPULSIS patients for whom data were available for at least one 3-month interval during the 12-month study period. No imputation was performed on missing values. Logistic regression was performed to identify associations between clinico-demographic factors and the primary outcomes of the number of hospitalisations or visits to a physician. First, a univariate analysis was used to examine associations between individual factors and outcomes. Factors that emerged as significant predictors in that analysis were then combined into a multivariate model using a backward selection algorithm. The regression procedure controlled for the fact that each patient contributed data to up to four 3-month intervals in the dataset, by allowing all observations relating to the same patient to be correlated. The uni- and multivariate models were used to identify factors significantly associated with the outcomes. The final multivariate models were also assessed for their sensitivity and specificity for predicting the primary outcomes. 
Table 1 INPULSIS and present study baseline characteristics

\begin{tabular}{llll}
\hline Characteristics & \multicolumn{2}{l}{ Pooled data of INPULSIS 1 and 2} & \multicolumn{2}{l}{\begin{tabular}{l} 
Present study \\
\cline { 2 - 3 }
\end{tabular}} & Placebo & Nintedanib $150 \mathrm{mg}$ bid & Both treatment arms (pooled) \\
\hline Number of patients & $423(100.0)$ & $638(100.0)$ & $1014(100.0)$ \\
Age (years) & $67.0 \pm 7.9$ & $66.6 \pm 8.1$ & $66.7 \pm 8.0$ \\
Sex & & & \\
Male & $334(79.0)$ & $507(79.5)$ & $805(79.4)$ \\
Female & $89(21.0)$ & $131(20.5)$ & $209(20.6)$ \\
Weight (kg) & $78.64 \pm 16.54$ & $79.23 \pm 16.57$ & $79.24 \pm 26.52$ \\
Height (cm) & $168.2 \pm 9.1$ & $167.5 \pm 9.3$ & $169.94 \pm 9.21$ \\
Smoking history & & & $282(27.8)$ \\
Never smoked & $122(28.8)$ & $174(27.3)$ & $689(67.9)$ \\
Ex-smoker & $283(66.9)$ & $435(68.2)$ & $43(4.2)$ \\
Currently smokes & $18(4.3)$ & $29(4.5)$ & $1.61 \pm 1.33$ \\
Time since diagnosis $($ years) & $1.57 \pm 1.31$ & $1.65 \pm 1.36$ & 0.0 \\
Mean \pm SD & 0.0 & 0.0 & 1.2 \\
Minimum & 1.17 & 1.26 & 5.0 \\
Median & 5.0 & 5.20 & $2734.59 \pm 772.93$ \\
Maximum & $2727.7 \pm 810.2$ & $2713.5 \pm 757.0$ & $78.3 \pm 18.2^{\mathrm{a}}$ \\
FVC (ml) & $79.27 \pm 18.22$ & $79.74 \pm 17.57$ & \\
FVC\%pred & & & \\
\hline
\end{tabular}

Data are presented as $N(\%)$ or mean $\pm \mathrm{SD}$

bid twice daily, $F V C$ force vital capacity, $F V C \%$ pred force vital capacity percent predicted, $S D$ standard deviation

${ }^{a}$ The value reflects the FVC\%pred at the beginning of any of the available 3-month intervals (3774)
Analyses of secondary outcomes were based on data from the subset of patients who experienced a primary outcome. These data were analysed using several modelling approaches: truncated Poisson regression, negative binomial regression, standard regression with or without log transformation, and logistic regression after converting duration of hospitalisation to a dichotomous variable ( $\leq 1$ or $>1$ week).

\section{Results}

The INPULSIS RCTs were double-blind, placebo-controlled studies involving 1066 patients at 205 sites in 24 countries in the Americas, Europe, Asia, and Australia. Patients were followed-up over a 12-month treatment regimen of nintedanib or placebo. The efficacy and safety results of the clinical trials are reported in detail elsewhere $[8,9]$.

Not all patients had available data to be analysed in the present study. This was the case with patients who died or were lost to follow-up before completion of the first 3-month interval in INPULSIS or for whom FVC\%pred data were incomplete (Fig. 1). The present study examined 1014 INPULSIS patients for whom sufficient data were available. The baseline characteristics for this group were similar to those of the entire randomised INPULSIS populations (Table 1). The mean age at baseline was $66.7 \pm 8.0$ years (range 42-89; median 67), and 805 $(79.4 \%)$ were men. Nearly two-thirds of the patients were ex-smokers $(67.9 \%)$, followed by never smokers $(27.8 \%)$ and current smokers (4.2\%).

Data were available for 843 patients for all four 3-month intervals during the study period, for 91 patients for three intervals, for 49 patients for two intervals and for 31 patients for only one interval (Fig. 2). Altogether, the dataset contained 3774 intervals of 3 months for the current-interval analysis. The previous-interval analysis included a subset of 2767 intervals, because there were no observations for the interval preceding 0-3 months.

Across all 3774 intervals, the average change observed in FVC\%pred was -1.1 percentage points (standard deviation [SD] 5.6 points). Of those, 2251 (59.6\%) were negative changes (drop in FVC\% \%red); a three-point drop occurred 1169 (31.0\%) times, a five-point drop 691 (18.3\%) times and a 10-point drop 175 (4.6\%) times. There were $35(0.9 \%)$ observed investigator-reported acute exacerbations. ${ }^{3}$

\footnotetext{
3 All exacerbation events were considered for the period from baseline until the day of the last FVC measure in the trial period for each patient (circa 12 months).
} 
843

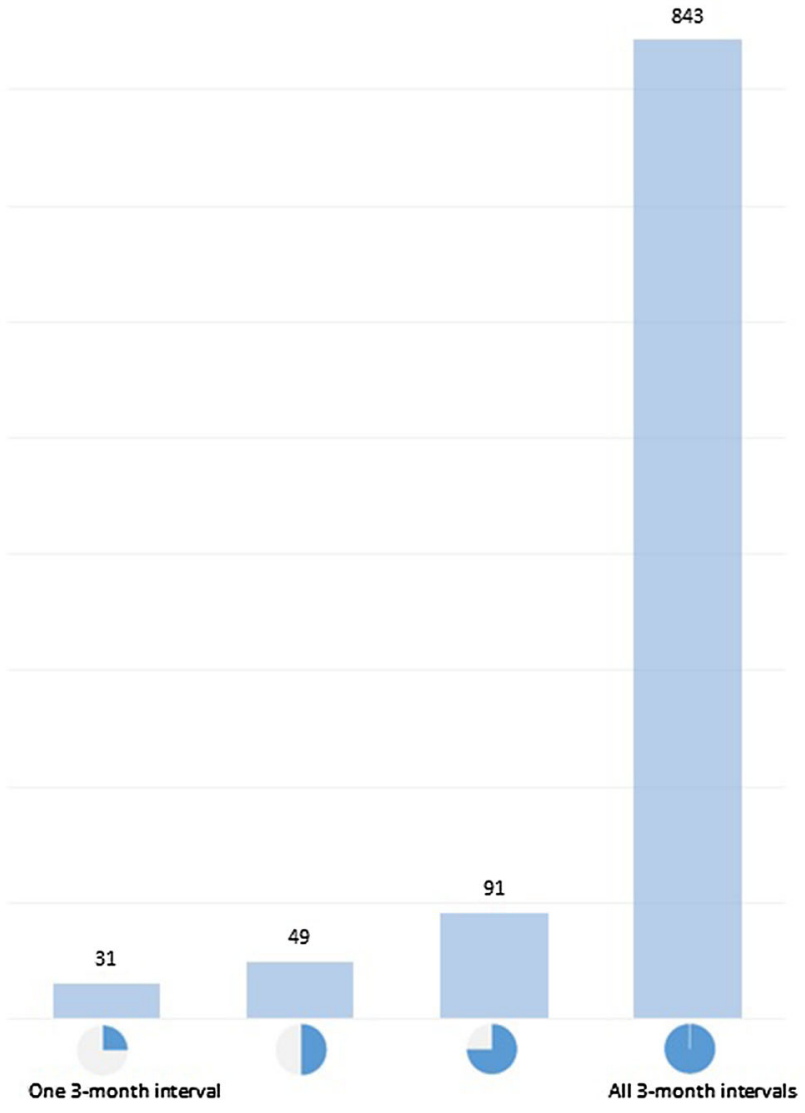

Fig. 2 Number of patients with available 3-month intervals for the analysis

The mean duration of hospitalisation was 10.6 days (SD 12.7; range 1-80; median 7) (Table 2). Visits to a physician occurred in 1136 of 3774 intervals (30.1\%). During these intervals, the mean number of visits was 2.0 (SD 1.8; range 1-22; median 1). The number of hospitalisation visits were similar between gender-age-physiology (GAP) index stages at baseline. The duration of hospitalisation was higher for patients with GAP index stage III at baseline (Table 3).

\subsection{Factors Associated with Hospitalisation}

A categorical analysis of absolute FVC\%pred change, acute exacerbations and hospitalisation suggested a strong relationship between the variables (Table 4). Further to this, various factors were examined for their possible association with the occurrence of at least one hospitalisation during a given 3-month interval (Table 5). Our analysis estimated that the odds of hospitalisation were 1.58 and 2.62 times higher for patients who had lost, respectively, 5 and 10 percentage points of FVC\%pred within the same 3-month interval. The odds of hospitalisation were more than 14 times higher when an acute exacerbation occurred. No other predictors were estimated as statistically significant. The above factors remained significant when repeating the analysis for hospitalisation based on change in FVC\% pred or events occurring during the previous 3-month interval. Smoker status and a unit change in $\mathrm{FVC} \%$ pred during the previous interval were added to the significant factors (Table 5).

As a second step, we combined the above variables into a multivariate model (see Table 6 for current-interval and for previous-interval analysis). On the current-interval analysis, the 5-point FVC\%pred drop variable was no longer statistically significant. The analysis showed that a loss of at least FVC10\%pred and the occurrence of an acute exacerbation during a 3-month interval were significantly associated with increased odds of being hospitalised during that same interval. The odds of being hospitalised during a given 3-month interval were 2.12 times higher for patients who had lost 10 points of $\mathrm{FVC} \%$ pred or more during that interval than for those who did not decline. The odds of being hospitalised during a given 3-month interval were 12.37 times higher for patients who experienced an acute exacerbation during that interval than for those who did not. The model did not change when we tried a $10 \%$ probability of significance level. When we combined the current and previous interval factors into a multivariate model, the odds remained similar for all significant variables (Table 7).

Table 2 Hospitalisation and FVC\%pred level at baseline

\begin{tabular}{lrrlr}
\hline $\begin{array}{l}\text { Baseline } \\
\text { FVC\%pred }\end{array}$ & Patients & Patients with hospitalisations & Hospitalisations per patient hospitalised & Duration of hospitalisation (days) \\
\hline$<50$ & $5(0.5)$ & $1(20.0)$ & $1 \pm-$ & $2 \pm-$ \\
$50-80$ & $544(53.6)$ & $84(15.4)$ & $1.2 \pm 0.4$ & $10.1 \pm 12.01$ \\
$>80$ & $465(45.9)$ & $69(14.8)$ & $1.2 \pm 0.5$ & $11.1 \pm 13.4$ \\
Overall & $1014(100)$ & $154(15.2)$ & $1.2 \pm 0.4$ & $10.6 \pm 12.7$ \\
\hline
\end{tabular}

Data are presented as $N(\%)$ or mean $\pm \mathrm{SD}$

$F V C \%$ pred force vital capacity percent predicted, $S D$ standard deviation 
Table 3 Hospitalisation and GAP index stage at baseline

\begin{tabular}{lclll}
\hline Baseline GAP index & Patients $^{\mathrm{a}}$ & Patients with hospitalisations & Hospitalisations per patient hospitalised & Duration of hospitalisation (days) \\
\hline Stage I & $493(48.6)$ & $65(13.2)$ & $1.2 \pm 0.5$ & $10.4 \pm 11.8$ \\
Stage II & $455(44.9)$ & $75(16.5)$ & $1.1 \pm 0.4$ & $10.1 \pm 12.0$ \\
Stage III & $65(6.4)$ & $14(21.5)$ & $1.2 \pm 0.4$ & $14.7 \pm 19.2$ \\
\hline
\end{tabular}

Data are presented as $N(\%)$ or mean $\pm \mathrm{SD}$

$D L_{C O}$ diffusing capacity of the lung for carbon monoxide $(\mathrm{CO}), G A P$ gender-age-physiology, $S D$ standard deviation

${ }^{\mathrm{a} O n e}$ patient had a missing $\mathrm{DL}_{\mathrm{CO}}$ reading, which was assumed to be missing at random, not that the patient could not perform the test

Table 4 FVC\%pred drop and acute exacerbations and hospitalisation

\begin{tabular}{lclc}
\hline & Number of 3-month time intervals & Hospitalisations reported $^{\text {a }}$ & Duration of hospitalisation (days) \\
\hline 3 percentage points drop in FVC\%pred & 1169 & $61(5.2)$ & $13.7 \pm 17.3$ \\
5 percentage points drop in FVC\%pred & 691 & $46(6.7)$ & $13.9 \pm 17.5$ \\
10 percentage points drop in FVC\%pred & 175 & $19(10.9)$ & $10.9 \pm 12.3$ \\
All 35 acute exacerbation events & 35 & $14(40)$ & $14.8 \pm 13.8$ \\
\hline
\end{tabular}

Data are presented as $N, N(\%)$ or mean $\pm \mathrm{SD}$

FVC\%pred force vital capacity percent predicted, $S D$ standard deviation

${ }^{a}$ The percentage refers to the number of hospitalisations reported out of the total events observed (change in FVC\%pred or acute exacerbations). For example: Hospitalisations were reported in $40 \%$ of the times an event occurred; that is, $14 / 35$

Table 5 Univariate analysis to identify factors associated with hospitalisation of patients with IPF during 3 months

\begin{tabular}{|c|c|c|c|c|c|}
\hline \multirow[t]{2}{*}{ Predictor parameter } & \multirow[t]{2}{*}{ Modality } & \multicolumn{2}{|c|}{ Current-interval analysis } & \multicolumn{2}{|c|}{ Previous-interval analysis ${ }^{\mathrm{a}}$} \\
\hline & & OR $(95 \% \mathrm{CI})$ & $p$ value & OR $(95 \% \mathrm{CI})$ & $p$ value \\
\hline FVC\%Pred at beginning of interval & For 10 -unit decrement & $1.08(0.97,1.21)$ & 0.169 & $1.06(0.95,1.20)$ & 0.305 \\
\hline Change in FVC\%Pred over interval & For 10 -unit decrement & $1.28(0.96,1.70)$ & 0.087 & $1.46(1.10,1.93)$ & 0.008 \\
\hline Any drop in $\mathrm{FVC} \%$ Pred during interval & Yes (vs no) & $1.08(0.80,1.47)$ & 0.611 & $0.99(0.71,1.39)$ & 0.976 \\
\hline $\begin{array}{l}\text { Drop of } 3 \text { points in FVC\%Pred during } \\
\text { interval }\end{array}$ & Yes (vs no) & $1.16(0.85,1.59)$ & 0.355 & $1.40(0.99,1.97)$ & 0.058 \\
\hline $\begin{array}{l}\text { Drop of } 5 \text { points in } \mathrm{FVC} \% \text { Pred during } \\
\text { interval }\end{array}$ & Yes (vs no) & $1.58(1.12,2.23)$ & 0.009 & $1.70(1.18,2.46)$ & 0.005 \\
\hline $\begin{array}{l}\text { Drop of } 10 \text { points in FVC\%Pred during } \\
\text { interval }\end{array}$ & Yes (vs no) & $2.62(1.53,4.49)$ & $<0.001$ & $3.22(1.87,5.53)$ & $<0.001$ \\
\hline Acute exacerbation during interval & Yes (vs no) & $14.44(7.31,28.52)$ & $<0.001$ & $3.47(1.06,11.38)$ & 0.040 \\
\hline Age at baseline & For 5-year increment & $1.08(0.97,1.21)$ & 0.151 & $1.08(0.96,1.21)$ & 0.222 \\
\hline Time since IPF diagnosis & For 1-year increment & $1.01(0.90,1.14)$ & 0.826 & $0.98(0.86,1.11)$ & 0.733 \\
\hline Gender & Female (vs male) & $1.27(0.85,1.92)$ & 0.247 & $1.25(0.81,1.93)$ & 0.320 \\
\hline \multirow[t]{4}{*}{ Smoking status at baseline } & Ex-smoker (vs never-smoker) & $1.33(0.89,1.99)$ & 0.159 & $1.38(0.91,2.09)$ & 0.126 \\
\hline & $\begin{array}{l}\text { Current smoker (vs never- } \\
\text { smoker) }\end{array}$ & $1.88(0.99,3.60)$ & 0.056 & $2.35(1.21,4.57)$ & 0.012 \\
\hline & Current smoker (vs others) & $1.52(0.86,2.70)$ & 0.148 & $1.85(1.02,3.35)$ & 0.042 \\
\hline & Never-smoked (vs others) & $0.73(0.49,1.09)$ & 0.123 & $0.70(0.46,1.05)$ & 0.083 \\
\hline
\end{tabular}

FVC\% Pred force vital capacity percent predicted; IPF idiopathic pulmonary fibrosis; $v s$ versus

${ }^{a}$ This analysis was conducted on a subset of the primary analysis (total of 2767 observation) since we have no evidence for the interval before the 0-3 months 
Table 6 Multivariate model for hospitalisations during a 3-month interval

\begin{tabular}{|c|c|c|c|c|}
\hline \multirow[t]{2}{*}{ Predictor } & \multicolumn{2}{|c|}{ Current-interval analysis } & \multicolumn{2}{|c|}{ Previous-interval analysis* } \\
\hline & OR $(95 \% \mathrm{CI})$ & $p$ value & OR $(95 \% \mathrm{CI})$ & $p$ value \\
\hline Drop of 10 points in FVC\% Pred during interval: yes vs no & $2.12(1.15,3.91)$ & 0.017 & $3.22(1.87,5.53)$ & $<0.001$ \\
\hline Exacerbation during interval: yes vs no & $12.37(5.92,25.89)$ & $<0.001$ & - & \\
\hline
\end{tabular}

OR odds ratio; $C I$ confidence interval; $F V C \%$ Pr0065d force vital capacity percent predicted; $v s$ versus

${ }^{a}$ This analysis was conducted on a subset of the primary analysis (total of 2767 observation) since we have no evidence for the interval before the 0-3 months. Note that exacerbation was not significant in the univariate analysis

Table 7 Multivariate model for hospitalisations during the previous 3-month interval and at the current 3-month interval

\begin{tabular}{lrr}
\hline Predictor & OR (95\% CI) & $p$ value \\
\hline Drop of 10 points in FVC\%pred during previous interval: yes vs. no & $3.44(2.01-5.88)$ & $<0.001$ \\
Drop of 10 points in FVC\%pred during current interval: yes vs. no & $2.11(1.10-4.05)$ & 0.025 \\
Exacerbation during current interval: yes vs. no & $12.13(5.25-28.04)$ & $<0.001$ \\
\hline
\end{tabular}

CI confidence interval, FVC\%pred force vital capacity percent predicted, OR odds ratio
The predictive power of the model (the percentage of observations correctly classified) was high (95.1\%). However, this was entirely driven by the fact that $95 \%$ of observations in the dataset were non-events. The best predictive value achieved was a sensitivity of $7.8 \%$ and a specificity of $99.4 \%$.

Attempts with various types of regression and non-regression modelling failed to identify significant predictors of specific duration of hospitalisation (data not shown).

\subsection{Factors Associated with Visits to a Physician}

Various factors were examined for their possible association with the occurrence of at least one visit to a physician during a given 3-month interval (Table 8). We found that the odds of having at least one visit during a given 3-month interval was 1.05 times higher for each difference of 10 points in FVC\%pred at the start of the interval and 1.13 times higher for each difference of 10 points in FVC\%pred during the interval. Moreover, a loss of 5 points in

Table 8 Univariate analysis to identify factors associated with physician visits of patients with IPF during 3 months

\begin{tabular}{|c|c|c|c|c|c|}
\hline \multirow[t]{2}{*}{ Predictor parameter } & \multirow[t]{2}{*}{ Modality } & \multicolumn{2}{|c|}{ Current-interval analysis } & \multicolumn{2}{|c|}{ Previous-interval analysis ${ }^{2}$} \\
\hline & & OR $(95 \% \mathrm{CI})$ & $p$ value & OR $(95 \% \mathrm{CI})$ & $p$ value \\
\hline FVC\%Pred at beginning of interval & For 10 -unit decrement & $1.05(1.00,1.10)$ & 0.040 & $1.04(0.99,1.10)$ & 0.141 \\
\hline Change in FVC\% Pred over interval & For 10 -unit decrement & $1.13(1.00,1.28)$ & 0.042 & $0.93(0.81,1.06)$ & 0.293 \\
\hline Any drop in $\mathrm{FVC} \%$ Pred during interval & Yes (vs no) & $1.10(0.95,1.26)$ & 0.197 & $0.90(0.77,1.05)$ & 0.178 \\
\hline Drop of 3 points in FVC\% Pred during interval & Yes (vs no) & $1.14(0.98,1.32)$ & 0.096 & $1.07(0.90,1.28)$ & 0.433 \\
\hline Drop of 5 points in FVC\% Pred during interval & Yes (vs no) & $1.23(1.03,1.46)$ & 0.020 & $1.05(0.85,1.30)$ & 0.638 \\
\hline Drop of 10 points in FVC\%Pred during interval & Yes (vs no) & $1.26(0.90,1.75)$ & 0.175 & $0.88(0.58,1.32)$ & 0.526 \\
\hline Acute exacerbation during interval & Yes (vs no) & $1.75(0.91,3.37)$ & 0.094 & $0.95(0.36,2.53)$ & 0.924 \\
\hline Age at baseline & For 5-year increment & $1.07(1.01,1.14)$ & 0.028 & $1.07(0.99,1.14)$ & 0.070 \\
\hline Time since IPF diagnosis & For 1-year increment & $1.04(0.97,1.12)$ & 0.244 & $1.03(0.95,1.12)$ & 0.427 \\
\hline Gender & Female (vs male) & $1.32(1.05,1.67)$ & 0.017 & $1.29(1.00,1.67)$ & 0.047 \\
\hline \multirow[t]{4}{*}{ Smoking status at baseline } & Ex-smoker (vs never-smoker) & $0.96(0.77,1.19)$ & 0.692 & $0.91(0.72,1.16)$ & 0.450 \\
\hline & Current smoker (vs never-smoker) & $0.94(0.59,1.50)$ & 0.809 & $0.85(0.49,1.46)$ & 0.545 \\
\hline & Current smoker (vs others) & $0.97(0.63,1.51)$ & 0.904 & $0.90(0.54,1.51)$ & 0.693 \\
\hline & Never-smoked (vs others) & $1.04(0.85,1.29)$ & 0.684 & $1.10(0.87,1.39)$ & 0.424 \\
\hline
\end{tabular}

FVC\% Pred force vital capacity percent predicted; IPF idiopathic pulmonary fibrosis; $v s$ versus

${ }^{a}$ This analysis was conducted on a subset of the primary analysis (total of 2767 observation) since we have no evidence for the interval before the 0-3 months 
Table 9 Multivariate model for physician visits during the current 3-month interval

\begin{tabular}{lll}
\hline Predictor & OR (95\% CI) & $p$ value \\
\hline Age at baseline: for 1-year increment & $1.02(1.00-1.03)$ & 0.012 \\
Sex: male vs. female & $0.73(0.58-0.92)$ & 0.008 \\
FVC\%pred at beginning of interval; for 1-unit increment & $0.99(0.99-1.00)$ & 0.005 \\
Drop of 5 points in FVC\%pred during interval: yes vs. no & $1.23(1.03-1.47)$ & 0.02 \\
\hline
\end{tabular}

CI confidence interval, FVC\%pred force vital capacity percent predicted, OR odds ratio
FVC\%pred during the interval increased the odds of a physician visit by 1.23 times. Age and sex were also associated with higher odds: the odds of having at least one visit during a given 3-month interval were 1.07 times higher for each increase of 5 years in age at baseline, and the odds of having at least one visit during a given 3-month interval were 1.32 times higher for female patients than for males. The other predictors we tested did not show statistical significance. When repeating the analysis for visits due to changes in FVC\%pred or events occurring during the previous interval, most factors were not statistically significant. Sex was the only significant factor (Table 8). For this reason, this analysis was not explored any further with multivariate modelling.

For the current-interval analysis, after combining the significant factors into a multivariate model, we maintained statistical significance in all variables except the FVC\% $\%$ red change during the interval (Table 9). The model did not change when we tried a $10 \%$ probability of significance level. The predictive value achieved by this model was a sensitivity of $55.7 \%$ and a specificity of $54.4 \%$, with an overall proportion of observations correctly classified of $54.8 \%$. This suggests only a marginal improvement compared with a model based on chance only and is therefore not a satisfactory predicting model for this outcome.

Similar to the hospitalisation healthcare resource use intensity analysis, attempts with various types of regression and non-regression modelling failed to identify significant predictors of the number of visits during a given 3-month interval (data not shown).

\section{Discussion}

This study provides insights into how IPF progression and acute exacerbations can affect hospitalisations and physician visits. The evidence base was a relatively large global patient population, drawn from two rigorous RCTs, which examined patients over 12 months of treatment. Since median survival of patients with IPF in the UK is 3 years after diagnosis and only $20 \%$ survive longer than 5 years [2], examination of the disease over 1 year should provide a reasonable basis for estimating healthcare use.
Our results suggest that the occurrence of acute exacerbations is associated with significantly more hospitalisations, implying increased use of in-hospital resources. However, acute exacerbations were not associated with increased visits to a physician. This may mean that acute exacerbations influence primarily hospitalisation-related costs, which is consistent with the often-severe nature of this complication and its strong association with morbidity and mortality [11-13]. Our analysis covered unscheduled visits to the hospital or physicians. It follows that if progression or acute exacerbations coincided with any study per-protocol visits, then our estimates are likely to underestimate the true impact of the disease on healthcare resource use.

Disease progression, as reflected by $\mathrm{FVC} \%$ pred status, was associated with an increase in both occurrence of hospitalisation and physician visits. A loss of 5 or 10 FVC\% \%red points in a 3-month interval was linked to a statistically significant increase in the odds of hospitalisation. The same binary analysis was repeated for the physician visits but was not found to be statistically significant. Instead, the condition of the patient at baseline and a discreet change per 1-unit of FVC\%pred were significant predictors of increase in physician visits.

To explore further the association between the events occurred and hospitalisation or physician visits, we conducted two analyses on events and changes from the previous and the current interval. The statistically significant factors with an impact on hospitalisation remained largely similar between the two analyses. When the variables were combined in a multivariate model, the odds of hospitalisation were also similar to when the previous and currentinterval analyses were run separately. The latter suggests that the impact would not change whether the change occurred in the past or present 3 months. The same was not observed when exploring significant factors associated with physician visits.

Although in theory the timing of the exacerbation events is easier to analyse in relation to hospitalisation or physician visits, the rareness of the events meant we did not have enough observations to form any conclusions.

Our objective was to analyse the INPULSIS-1 and -2 healthcare use evidence to provide estimates of association with several patient characteristics and disease progression. 
Although some associations were found to be statistically significant, the results fell short of providing meaningful predictive outcomes. On both occasions, when a multivariate model was used to combine all the significant univariate variables, the predictive value of the model was found to be poor. Further examination of the resource use intensity (length of stay and number of visits) was also unsuccessful. This is likely to be due to insufficient data to show an acceptable level of significance. For example, FVC\%pred negative changes within a 3-month interval (disease progression) were only $18 \%$ (5-point) and 5\% (10point) of all the observed changes in this variable. The number of exacerbations observed in the trial were too few (35 events) to allow any further analysis. The lack of significance may also be because the events have an unpredictable impact on the duration of hospitalisation or the number of physician visits, and it is difficult to determine a trend.

It is an important limitation of our analysis that our selection of covariates was not exhaustive. We were primarily guided by the conceptualisation of disease models in the literature that used FVC\%pred and, at times, acute exacerbations to reflect natural progression [17]. The analysis did not control for seasons and climate. As these were not variables collected in the clinical trials, implementation would require assumptions based on the locations of the investigation centres around the world.

Another limitation of the analysis is that all estimates were based on two experimental drug clinical trials [9]. Although such studies are considered the gold standard to evaluate the comparative efficacy and safety of new interventions, their use may not be suitable for drawing conclusions on healthcare resource use. The resource use from the clinical trial may not be a good representation of real-world use: the presence of a protocol for patient monitoring and frequent patient access to healthcare professionals may underestimate visits for nonurgent issues. Further, hospitalisation practice may differ between healthcare systems, which would not be reflected in a multinational clinical trial because of the pooling of the data. Moreover, the resource use evidence was a further outcome in both studies, and reporting is likely to have been less rigorous compared with the primary clinical outcomes.

No data imputation was performed for patients lost to follow-up during the observation period or with missing information. As a comparison, we obtained 3774 intervals of 3 months from the clinical trials. Of those, 843 patients had data for four intervals, 91 patients had data for three intervals, 49 patients had data for two intervals and 31 patients had data for only one interval. Data imputation in most cases would require assumptions on the FVC\% pred at the start or end of the intervals, which is difficult to predict. Moreover, by imputing all the missing data, we would get a maximum of 4056 data; an increase of $7.5 \%$ in complete data intervals. Overall, the impact of not imputing missing values was deemed to be minimal to the final model results.

Our analysis considered all INPULSIS patients, including those receiving active treatment with nintedanib. Given that acute exacerbation events were rare in the clinical trials, by pooling all trial arms we reduced the chance of a type 2 error in our results (non-significance because of a small sample). Our research question was about the impact of the patient condition, not the treatment effect, on resource use. The effect of adding treatment as a covariate in the models was not statistically significant, and the final models remained unchanged.

Several recent studies suggested that IPF incurred a substantial economic and health burden [18-23]. The methods varied between studies from an Adelphi panel [22], database analyses of patients with IPF [20, 21] and comparisons of IPF and non-IPF estimates [18, 19, 23]. The latter provide useful information on the relative cost burden of IPF from a US-payer perspective, and both studies agreed on a more than twofold increase in the annual rate of hospitalisation (post-index) [18, 19, 23]. Raimundo et al. [20] estimated that respiratory events were responsible for half of the hospitalisations that occurred during the 3 years of observations for patients with IPF. Although it is difficult to compare with accuracy with the other studies, our findings point to the same direction with increased odds of hospitalisation after a 10-point reduction in FVC\%pred during a 3-month interval. The Adelphi panel of clinical experts in Spain suggested that patients use considerable healthcare resources during an acute exacerbation and follow-up, including medical visits, hospitalisations and multiple tests [22]. This is also in line with our estimates of an increase in hospitalisation with acute exacerbation events. However, we did not find statistical significance in the increase in healthcare professional visits with each exacerbation event. This variation may be due to the aforementioned data limitations.

The present analysis helps lay the groundwork for more extensive modelling of healthcare resource use by patients with IPF. Such work will be valuable to those making decisions about resource use and allocation for patients with IPF. Such work will also help inform and guide future efforts at assessing and comparing treatments for the disease, which is critical given the limited range of drugs and alternative treatments available, including lung transplantation, oxygen therapy, and pulmonary rehabilitation $[2,10]$.

\section{Conclusion}

We found significant association between hospitalisation and both the loss in FVC\%pred and occurrence of exacerbation in 3 months. Furthermore, physician visits were 
significantly associated with the baseline FVC\%pred, a drop in FVC\%pred within a 3-month interval, patient baseline age and sex. However, the synthesis of these variables in a multivariate model could not accurately predict the occurrence of hospitalisation or visits.

Acknowledgements The authors would like to thank Chapin Rodriguez, Ph.D., for assisting with preparation of the manuscript.

Author Contributions CLR and AD conducted the analysis and wrote the manuscript. NS, DE and TM provided feedback on the analysis results and drafts of the manuscript. All authors approved the final draft of the manuscript.

Data Availability Statement The data that support the findings of this study are available from Boehringer Ingelheim International $\mathrm{GmbH}$, but restrictions apply to the availability of these data, which were used under license for the current study, and so are not publicly available. However, data are available from the authors upon reasonable request and with permission of Boehringer Ingelheim International $\mathrm{GmbH}$.

\section{Compliance with Ethical Standards}

The INPULSIS- 1 and INPULSIS- 2 clinical trials were carried out in compliance with the clinical trial protocols, the principles laid down in the Declaration of Helsinki, in accordance with the International Conference on Harmonisation (ICH) Harmonised Tripartite Guideline for Good Clinical Practice (GCP) and in accordance with applicable regulatory requirements and Boehringer Ingelheim (BI) standard operating procedures.

Informed consent Informed consent was obtained from all individual participants included in the study.

Funding This study was funded by Boehringer Ingelheim International $\mathrm{GmbH}$.

Conflict of interest Alex Diamantopoulos is employed by Symmetron Limited, which received funding from Boehringer Ingelheim International $\mathrm{GmbH}$ for this project. Alex Diamantopoulos has previously received funding from Boehringer Ingelheim for contributions to original research and similar articles. Toby M. Maher has received consulting fees from Symmetron Limited; industry-academic research funding from GlaxoSmithKline R\&D and UCB; and consultancy or speaker's fees from AstraZeneca, Bayer, Biogen Idec, Boehringer Ingelheim, Cipla, Dosa, Galapagos, GlaxoSmithKline R\&D, ProMetic, Roche (and previously InterMune), Sanofi-Aventis, Takeda and UCB. Toby M. Maher is supported by an NIHR Clinician Scientist Fellowship (NIHR ref: CS-2013-13-017) and British Lung Foundation Chair in Respiratory Research (C17-3). Nils Schoof and Dirk Esser are employees of Boehringer Ingelheim International $\mathrm{GmbH}$. Corinne LeReun has received consulting fees from Symmetron Limited.

Open Access This article is distributed under the terms of the Creative Commons Attribution-NonCommercial 4.0 International License (http://creativecommons.org/licenses/by-nc/4.0/), which permits any noncommercial use, distribution, and reproduction in any medium, provided you give appropriate credit to the original author(s) and the source, provide a link to the Creative Commons license, and indicate if changes were made.

\section{References}

1. Raghu G, Collard HR, Egan JJ, et al. An official ATS/ERS/JRS/ ALAT statement: idiopathic pulmonary fibrosis: evidence-based guidelines for diagnosis and management. Am J Respir Crit Care Med. 2011;183:788-824.

2. National Institute for Health and Care Excellence. Idiopathic pulmonary fifibrosis in adults: diagnosis and management. Clinical guideline 163; 2013. https://www.nice.org.uk/guidance/ cg163/resources/idiopathic-pulmonary-fibrosis-in-adults-diagnosi s-and-management-pdf-35109690087877. Accessed 4 June 2018.

3. Hutchinson J, Fogarty A, Hubbard R, et al. Global incidence and mortality of idiopathic pulmonary fibrosis: a systematic review. Eur Resp J. 2015;46:795-806.

4. King TEJ, Pardo A, Selman M. Idiopathic pulmonary fibrosis. Lancet. 2011;378:1949-61.

5. European Medicines Agency. CHMP assessment report Esbriet. Procedure No. EMEA/H/C/002154. http://www.ema.europa.eu/ docs/en_GB/document_library/EPAR_-_Public_assessment_repo rt/human/002154/WC500103073.pdf. Accessed 4 June 2018.

6. European Medicines Agency. CHMP assessment report Ofev. Procedure No. EMEA/H/C/003821/0000. http://www.ema. europa.eu/docs/en_GB/document_library/EPAR_-_Public_assess ment_report/human/003821/WC500182476.pdf. Accessed 04 June 2018 .

7. US Food and Drug administration. https://www.accessdata.fda. gov/drugsatfda_docs/appletter/2014/022535Orig1s0001tr.pdf. Ac cessed 4 June 2018.

8. Richeldi L, Costabel U, Selman M, et al. Efficacy of a tyrosine kinase inhibitor in idiopathic pulmonary fibrosis. N Engl J Med. 2011;365:1079-87.

9. Richeldi L, du Bois RM, Raghu G, et al. Efficacy and safety of nintedanib in idiopathic pulmonary fibrosis. $\mathrm{N}$ Engl J Med. 2014;370:2071-82.

10. Xaubet A, Ancochea J, Bollo E, et al. Recommendations of SEPAR: guidelines for the diagnosis and treatment of idiopathic pulmonary fibrosis. Arch Bronconeumol. 2013;49:343-53.

11. Song JW, Hong SB, Lim CM, et al. Acute exacerbation of idiopathic pulmonary fibrosis: incidence, risk factors and outcome. Eur Respir J. 2011;37:356-63.

12. Judge EP, Fabre A, Adamali HI, et al. Acute exacerbations and pulmonary hypertension in advanced idiopathic pulmonary fibrosis. Eur Respir J. 2012;40:93-100.

13. Collard HR, Ryerson CJ, Corte TJ, et al. Acute exacerbation of idiopathic pulmonary fibrosis. An international working group report. Am J Respir Crit Care Med. 2016;194:265-75.

14. Bjoraker JA, Ryu JH, Edwin MK, et al. Prognostic significance of histopathologic subsets in idiopathic pulmonary fibrosis. Am J Respir Crit Care Med. 1998;157:199-203.

15. Vancheri C, du Bois RM. A progression-free end-point for idiopathic pulmonary fibrosis trials: lessons from cancer. Eur Respir J. 2013;41:262-9.

16. StataCorp. Stata statistical software: release 14. College Station: StataCorp LP; 2015.

17. Rinciog C, Watkins M, Chang S, et al. A cost-effectiveness analysis of nintedanib in idiopathic pulmonary fibrosis in the UK. Pharmacoeconomics. 2016;35:479-91.

18. Wu N, Yu Y, Chuang C, et al. Healthcare resource utilization among patients diagnosed with idiopathic pulmonary fibrosis in the United States. J Med Econ. 2015;18:249-57.

19. Yu Y, Wu N, Chuang C, et al. Patterns and economic burden of hospitalizations and exacerbations among patients diagnosed with idiopathic pulmonary fibrosis. J Manag Care Spec Pharm. 2016;22:414-23. 
20. Raimundo K, Chang E, Broder MS, et al. Clinical and economic burden of idiopathic pulmonary fibrosis: a retrospective cohort study. BMC Pulm Med. 2016;16:2.

21. Pedraza-Serrano F, Lopez de Andres A, Jimenez-Garcia R, et al. Retrospective observational study of trends in hospital admissions for idiopathic pulmonary fibrosis in Spain (2004-2013) using administrative data. BMJ Open. 2017;7:e013156.
22. Morell F, Esser D, Lim J, et al. Treatment patterns, resource use and costs of idiopathic pulmonary fibrosis in Spain-results of a Delphi Panel. BMC Pulm Med. 2016;16:7.

23. Collard HR, Chen SY, Yeh WS, et al. Health care utilization and costs of idiopathic pulmonary fibrosis in US. Medicare beneficiaries aged 65 years and older. Ann Am Thorac Soc. 2015;12:981-7. 